\title{
ВАЛИДАЦИЯ МЕТОДИКИ КОМПЛЕКСНОЙ ОЦЕНКИ ПОЛИМОРБИДНОСТИ
}

\author{
(С) Журавлев Ю.И., Тхорикова В.Н.
}

\author{
Кафедра госпитальной терапии \\ Белгородского государственного национального исследовательского университета, Белгород \\ E-mail: leriks@,bk.ru
}

\begin{abstract}
Методика комплексной оценки полиморбидности (МКОП) разработана с целью совершенствования лечебнодиагностических процессов в условиях первичной медико-санитарной помощи. Валидация МКОП осуществлена в ходе клинического исследования пациентов с метаболическим синдромом на фоне полиморбидности. Внешняя и содержательная валидности были оценены с помощью опроса практикующих врачей и специалистов-экспертов. Надежность подтверждена путем оценки внутреннего постоянства и воспроизводимости. Конкурентная валидность подтверждена наличием сильной обратной связи индексов МКОП и результатов наиболее распространенных методик ее оценки. Чувствительность МКОП была доказана путем расчета предсказуемых различий ее индексов. В результате валидации МКОП доказаны ее внешняя, содержательная, конструктивная и критериальная валидности, надежность и чувствительность. МКОП рекомендуется к использованию в медицинских организациях, оказывающих первичную медико-санитарную помощь.
\end{abstract}

Ключевые слова: методика оценки полиморбидности, метаболический синдром, полиморбидность, первичная медико-санитарная помощь.

\section{VALIDATION OF THE METHOD OF COMPLEX EVALUATION OF POLYMORBIDITY Zhuravlev Y.I., Tkhorikova V.N.}

Department of Hospital Therapy of Belgorod National Research University, Belgorod

The method for the complex evaluation of polymorbidity (MCEP) is designed to improve the treatment and diagnostic processes in the context of primary health care. Validation of the MCEP was carried out during a clinical study of patients with a metabolic syndrome against the background of polymorbidity. External and substantive validity was assessed using a survey of practicing physicians and experts. Reliability is confirmed by assessing internal consistency and reproducibility. Criterial validity is confirmed by the strong feedback of the indices of the MCEP and the results of the most common methodologies for its evaluation. The sensitivity of the MCEP was proved by calculating predictable differences in its indices. As a result of the validation of the MCEP, its external, substantial, constructive, and criterial validity, reliability and sensitivity are proved. The MCEP is recommended for use in medical organizations providing primary health care.

Keywords: method for the evaluation of polymorbidity, metabolic syndrome, polymorbidity, primary health care.

Полиморбидность (П) - широко распространенное явление в терапевтической практике. С целью оптимизации и совершенствования лечебно-диагностической, реабилитационной, экспертной и профилактической работы с пациентами этой категории в условиях первичной медикосоциальной помощи (ПМСП) разработана методика комплексной оценки полиморбидности (МКОП). В основу методики положены принципы полипараметрического анализа и доказательной медицины. Ее суть заключается в одновременной регистрации анамнестических, клинических, лабораторных, инструментальных, функциональных, психологических и социальных параметров здоровья пациента, которые служат индикаторами, используемыми в математических расчетах. Индикаторы здоровья пациента для удобства использования разделены на 5 кластеров. В первый кластер вошли индикаторы, ассоциированные с немодифицируемыми параметрами здоровья, во второй - с модифицируемыми, в третий - наиболее распространенные сердечно- сосудистые синдромы и нозологические формы, в четвертый - другие заболевания и в пятый функциональные параметры. Конечные результаты МКОП вычислялись с использованием математических методов, представлялись как индекс полиморбидности (ИП) и промежуточные индексы (индекс немодифицируемых индикаторов здоровья (И1), индекс модифицируемых индикаторов здоровья (И2), индекс коморбидной патологии (ИЗ), индекс мультиморбидной патологии (И4), функциональный индекс (И5)) и выражались в условных единицах. ИП принимал значения в диапазоне от 0 до 1,0 у.е. Значение 0 у.е. соответствовало П, несовместимой с жизнью; 1,0 у.е. - отсутствию П. Промежуточные результаты по отдельным кластерам принимали значения в диапазоне $0-1,0$ у.е. Значение 1,0 у.е. соответствовало отсутствию патологического влияния на здоровье пациента индикаторов данного кластера, 0 у.е. - максимально возможному патологическому влиянию. На основе вычислений предложена методика качественной градации степе- 
ни П, которая легла в основу оптимизации ряда лечебно-диагностических, профилактических, экспертных, прогностических и реабилитационных процессов. Значения в диапазоне 1,0-0,80 у.е. соответствовали низкому уровню П; 0,79-0,50 у.е. - среднему уровню П и 0,49-0 у.е. - высокому уровню П.

Целью настоящего исследования была валидация МКОП - доказательство надежности, чувствительности, и следующих видов валидности (B): внешней, содержательной, конструктивной и критериальной.

\section{МАТЕРИАЛЫ И МЕТОДЫ ИССЛЕДОВАНИЯ}

Рандомизированное контролируемое проспективное клиническое исследование выполнялось на базе кафедры госпитальной терапии Белгородского государственного национального исследовательского университета, Белгородской областной клинической больницы Святителя Иоасафа и Городской больницы № 2 г. Белгорода в соответствии с этическими принципами Хельсинской декларации Всемирной Медицинской Ассоциации (Форталеза, Бразилия, октябрь 2013 г.), правилами GCP [2] и нормативно-правовыми документами Минздрава РФ.

Для решения поставленных задач было обследовано 110 пациентов, из них 47 мужчин $(42,7 \%)$ и 63 женщины $(57,27 \%)$, проживающих в г. Белгороде и Белгородском районе. Критериями включения в исследование послужили возраст 18 лет и старше, наличие информированного согласия, ІІІа группа состояния здоровья по результатам диспансеризации [4], наличие метаболического синдрома (MC), в том числе - осложненного развитием атеросклероза и сахарного диабета (СД) [6], наличие полиморбидной патологии (одновременное наличие у пациента двух и более нозологических форм).

Медиана возраста обследованных пациентов составила 58 (53-63) лет ${ }^{1}$ и колебалась в диапазоне 24-84 лет. Гипертонической болезнью (ГБ) страдали подавляющее большинство пациентов (98,2\%, 108/110). У трети пациентов наблюдался СД $(27,3 \%, 30 / 110)$. У каждого пятого пациента $(22,7 \%, 25 / 110)$ наблюдались последствия сердечно-сосудистых событий в виде постинфарктного кардиосклероза. Перенесенные мозговой инсульт (МИ) или транзиторная ишемическая атака (ТИА) выявлялись в каждом шестом случае (16,4\%, 18/110). Девять обследованных пациентов (8,1\%, 9/110) перенесли коронарную реваскуляризацию.

${ }^{1}$ Здесь и далее в тексте, если не указан другой способ, данные представлены в виде медианы и значений верхнего и нижнего квартилей Мe (Q1-Q3)
Более чем в половине случаев (55,5\%, 61/110) имелась хроническая сердечная недостаточность.

Bce пациенты имели полиморбидную патологию. Наиболее частыми являлись заболевания органов дыхания $(69,1 \%, 76 / 110)$, костномышечной $(69,1 \%, 76 / 110)$ и пищеварительной систем $(63,6 \%, 70 / 110)$. Заболеваниями суставов страдала без малого половина пациентов (45,5\%, 50/110). Нередко $(51,8 \%, 57 / 110)$ выявлялись болезни глаза и его придаточного аппарата. В трети случаев $(40,9 \%, 45 / 110)$ наблюдались болезни мочеполового аппарата.

На протяжении 6 месяцев пациенты, включенные в исследование, наблюдались по программе диспансерного наблюдения (ДН) в соответствии с приказом Министерства здравоохранения РФ «Об утверждении Порядка проведения диспансерного наблюдения» [5]. Всем пациентам, включенным в исследование, было проведено трехкратное обследование: после включения в исследование (первый этап), через 1 неделю (второй этап) и через 6 месяцев ДН (третий этап). Программа обследования включала общеклиническое обследование (физикальный осмотр по классической общепринятой методике, определение веса, роста, индекса массы тела, объема талии, лодыжечно-плечевого индекса); лабораторное обследование (клинический анализ крови, клинический анализ мочи, биохимический анализ крови с определением глюкозы натощак, креатинина, гликозилированного гемоглобина, общего холестерина (ОХС), холестерина липопротеинов низкой и высокой плотности, триглицеридов, мочевой кислоты, пероральный глюкозотолерантный тест, расчет скорости клубочковой фильтрации по формуле CKD-EPI, определение мочевой экскреции альбумина за сутки); инструментальное обследование (электрокардиография, эхокардиография, ультразвуковое исследование брахиоцефальных артерий, тредмил-тест, суточное мониторирование ЭКГ и АД, пульсоксиметрия); консультации специалистов (офтальмолог, невролог, гинеколог, уролог, кардиолог, отоларинголог, гастроэнтеролог, хирург, ревматолог, эндокринолог, уролог, психотерапевт). При наличии у пациента противопоказаний к нагрузочному тестированию, толерантность к физической нагрузке (ТФН) определялась при помощи индекса DASI [11]. Дополнительное обследование пациентов включало определение степени никотиновой зависимости (тест Фагерстрома) [9], оценку уровня тревоги и депрессии (HADS) [16], определение биологического возраста по методике В.П. Войтенко [1], качества жизни (SF-36) [16], риск сердечно-сосудистых осложнений при плановых оперативных вмешательствах [13]. По общепринятой методике определялся прогноз забо- 
левания, а также показания и противопоказания к санаторно-курортному лечению на основании приказа Минздравсоцразвития России [3].

Оценка П производилась с помощью МКОП и наиболее распространенных в доступной литературе методов ее оценки: система CIRS-G (Cumulative Illness Rating Scale for Geriatrics) [14], индекс Kaplan-Feinstein [12], индекс Charlson [7] с последующей оценкой степени корреляции результатов.

Внешняя и содержательная В изучались с помощью опроса специалистов - 10 врачейтерапевтов участковых со стажем работы не менее 10 лет и экспертов - научных работников (7 докторов и 2 кандидата медицинских наук). Надежность МКОП подтверждали оценкой внутреннего постоянства (вычисление коэффициента Кронбаха $\alpha$ [8]) и воспроизводимости (метод «тест-ретест»). Для оценки конструктивной В МКОП применяли количественные и качественные внешние критерии. Изучали их корреляцию с индексами МКОП и анализ сопряженности с качественной градацией степени П. Для доказательства прогностической критериальной В были исследованы сопряженности градаций ИП для стратификации признаков, имеющих прогностическое практическое значение при оказании ПМСП и их качественные значения, определенные общепринятыми методами. Оценка чувствительности МКОП осуществлялась на третьем этапе исследования путем расчета предсказуемых различий ее индексов в исследуемых группах. Были вычислены индексы чувствительности Guyatt Responsiveness Index (GRI) [10] для ИП и промежуточных индексов кластеров МКОП на первом (до начала ДН) и третьем (через 6 месяцев ДН) этапах исследования. Для этого были сформированы две группы: группа со стабильным состоянием здоровья пациентов, где изменения не ожидались, и группа с состоянием здоровья, претерпевшем значимые изменения со временем. В качестве критерия, отражающего изменение состояния здоровья пациентов с полиморбидной патологией на фоне МС, была избрана ТФН. Из 99 пациентов, выполнивших тест с дозированной физической нагрузкой в рамках третьего этапа исследования, были сформированы две группы: первая группа, численностью 29 человек, у которых ТФН повысилась более 2,0 METs в сравнении с исходным уровнем, и вторая группа (группа контроля), численностью 32 человека, изменение уровня ТФН у которых не превысило 1 METs. Индекс чувствительности GRI вычислялся как отношение среднего изменения значений в первой группе к стандартному отклонению изменений во второй группе, где изменения не ожидались.
Исследование взаимосвязи между парами дискретных качественных признаков проводилось с использованием анализа парных таблиц сопряженности. Помимо оценок критерия Пирсона Хи-квадрат $\left(\chi^{2}\right)$ и достигнутого уровня статистической значимости этого критерия, вычислялись и оценки интенсивности связи анализируемых признаков, такие как V-коэффициент Крамера. Корреляцию оценивали при помощи коэффициента Спирмена $\left(\mathrm{r}_{\mathrm{s}}\right)$, критическое значение уровня статистической значимости (p) принималось равным 0,05. Для сравнения центральных параметров групп использовались параметрические и непараметрические методы: дисперсионный анализ, в том числе с критерием КраскелаУоллиса и ранговыми метками Вилкоксона, медианный критерий и критерий Ван дер Вардена. Процедуры статистического анализа выполнялись с помощью статистических пакетов SAS 9.4, STATISTICA 12 и IBM-SPSS-23.

\section{РЕЗУЛЬТАТЫ ИССЛЕДОВАНИЯ И ИХ ОБСУЖДЕНИЕ}

В процессе опроса 10 врачей терапевтовучастковых (стаж работы не менее 10 лет) респонденты указали на отдельные недостаточно конкретизированные формулировки индикаторов. Результатом установления внешней В стала корректировка формулировок семи индикаторов.

Для доказательства содержательной В проведена экспертиза на предмет соответствия содержания индикаторов и кластеров МКОП содержанию и структуре понятия П, итогом которой стала корректировка формулировки 21 индикатора и сокращение их общего количества в реестре до 55, одобренных экспертами.

Надежность МКОП подтверждали оценкой внутреннего постоянства и воспроизводимости (таблица 1). Внутреннее постоянство определяли путем вычисления коэффициента Кронбаха $\alpha$ для каждого кластера отдельно по причине их разнородности [8]. Воспроизводимость подтверждали методом «тест-ретест» - оценка корреляции между первичным и повторным (через одну неделю) средними значениями ИП и промежуточных индексов у 86 пациентов, состояние здоровья которых по оценке исследователя на момент повторного обследования не изменилось. Среднее значение ИП в этой группе при первичном исследовании составило 0,69 $\pm 0,12$, при повторном $0,70 \pm 0,14^{2}$ у.е. Коэффициент корреляции Спирмена составил 0,86 ( $<0,0001)$. Для всех кластеров МКОП коэффициент Кронбаха $\alpha$ превысил 0,70,

2 Данные представлены в виде среднего значения и стандартного отклонения $(\mathrm{m} \pm \sigma)$. 
что подтверждает внутреннее постоянство кластеров МКОП. Установлена умеренная или сильная степень корреляции значений промежуточных индексов МКОП и высокая степень корреляции значений ИП методом «тест-ретест», что доказывает воспроизводимость МКОП.

Конструктивную В оценивали по ее конвергентной и дискриминативной характеристикам. Для этого с помощью коэффициента Спирмена $\left(\mathrm{r}_{\mathrm{s}}\right)$ проводилась оценка корреляции индексов МКОП с такими внешними критериями, как шкалы методики оценки качества жизни SF-36 [15], значение фактического биологического возраста (ФБВ), определенное методом И.П. Войтенко (1979) [1], количество сопутствующих заболеваний, уровни ОХС и систолического артериального давления (сАД), ТФН (таблица 2). Установлена связь признака ИП со всеми шкалами методики оценки качества жизни SF-36: сильная прямая $\left(\mathrm{r}_{\mathrm{s}}\right.$ в диапазоне 0,60-0,82, p<0,0001) - со шкалами, относящимися к физическому компоненту здоровья $(\mathrm{PH})$ и средняя по силе $\left(\mathrm{r}_{\mathrm{s}}\right.$ в диапазоне 0,500,76, $\mathrm{p}<0,0001)$ - со шкалами психологического компонента здоровья (МН), что соответствует гипотезе. Были статистически подтверждены гипотезы о наличии и отсутствии различных по силе корреляций промежуточных индексов МКОП с внешними критериями. Были подтверждены ожидания высокого уровня корреляции ИП и, особенно, $И_{4}$ с признаком количества сопутствующих заболеваний: $\mathrm{r}_{\mathrm{s}}$ составил 0,71 и 0,89 ( $\left.<<0,0001\right)$ соответственно. Были доказаны гипотезы: сильная связь признака И5 со шкалами SF-36, отражающими физическое функционирование, средняя по силе связь И5 со шкалами, отражающими эмоциональное функционирование и слабая связь со шкалами BP, GH, VT и MT. Также соответствуют гипотетическим ожиданиям сильная связь ряда промежуточных индексов с одними внешними признаками (И1 и ФБВ, И2 и уровень АД,
И2 и уровень ОХС, ИЗ и ТФН) и отсутствие или слабая интенсивность связи промежуточных индексов с другими внешними показателями (И1 и уровень АДс, И2 и количество сопутствующих заболеваний, И3 и количество сопутствующих заболеваний, И4 и уровень ОХС, И5 и уровень $\mathrm{OXC})$.

Одним из результатов МКОП является качественная градация степени П в соответствии с полученным ИП. Для обоснования конструктивной В этой градации был проведен анализ сопряженности этого признака с внешними качественными критериями (таблица 3). Обнаружена статистически значимая взаимосвязь степени П и факта перенесенных инфаркта миокарда и ОНМК, ТИА в прошлом, стадии ГБ, ХСН и ХБП, наличия тяжелых осложнений СД, степени тяжести заболеваний дыхательной и костно-мышечной систем. По интенсивности связь находится на среднем уровне (V-критерий Крамера в диапазоне 0,29$0,50)$. В то же время не выявлено статистически достоверной связи степени П и пола. Анализ сопряженности степени П и вышеприведенных качественных признаков доказывает конвергентную и дикриминативную В качественной градации степеней П на основании результатов МКОП.

Для оценки критериальной текущей (или конкурентной) В была использована связь ИП с итоговыми результатами других известных методов оценки П: индекс Kaplan-Feinstein, система CIRS$\mathrm{G}$, индекс Charlson. Коэффициент корреляции Спирмена составил: -0,90, -0,91 и -0,83 соответственно (уровень значимости $\mathrm{p}<0,0001$ ). Таким образом, обнаружена сильная обратная связь результатов оценки П посредством МКОП и результатов других наиболее распространенных методик ее оценки, что доказывает критериальную В МКОП.

Оценка внутреннего постоянства и воспроизводимости промежуточных индексов

Таблица 1 методики комплексной оценки полиморбидности

\begin{tabular}{|l|c|c|}
\hline \multicolumn{1}{|c|}{ Кластеры МКОП } & $\begin{array}{c}\text { Коэффициент } \\
\text { Кронбаха } \alpha\end{array}$ & $\begin{array}{c}\text { Коэффициент корреляции } \\
\text { Спирмена («тест-ретест»), }<0,0001\end{array}$ \\
\hline Немодифицируемые индикаторы здоровья & 0,74 & 0,86 \\
\hline Модифицируемые индикаторы здоровья & 0,86 & 0,74 \\
\hline $\begin{array}{l}\text { Коморбидная патология } \\
\text { (сердечно-сосудистые синдромы и нозологии) }\end{array}$ & 0,87 & 0,65 \\
\hline Мультиморбидная патология (другие нозологии) & 0,84 & 0,71 \\
\hline Функциональный статус & 0,79 & 0,68 \\
\hline
\end{tabular}


Оценка корреляции индексов методики комплексной оценки полиморбидности с количественными внешними критериями

\begin{tabular}{|c|c|c|c|c|c|c|}
\hline Внешние критерии & $\begin{array}{c}\text { Индекс } \\
\text { полимор- } \\
\text { бидности } \\
\text { (ИП) }\end{array}$ & $\begin{array}{c}\text { Индекс } \\
\text { немодифи- } \\
\text { цируемых } \\
\text { индикаторов } \\
\text { здоровья } \\
\text { (И1) }\end{array}$ & $\begin{array}{c}\text { Индекс } \\
\text { модифици- } \\
\text { руемых } \\
\text { индикаторов } \\
\text { здоровья } \\
\text { (И2) }\end{array}$ & $\begin{array}{c}\text { Индекс } \\
\text { комор- } \\
\text { бидной } \\
\text { патоло- } \\
\text { гии (И3) }\end{array}$ & $\begin{array}{c}\text { Индекс } \\
\text { мульти- } \\
\text { морбидной } \\
\text { патологии } \\
\text { (И4) }\end{array}$ & $\begin{array}{c}\text { Функ- } \\
\text { циональ- } \\
\text { ный } \\
\text { индекс } \\
\text { (И5) }\end{array}$ \\
\hline $\begin{array}{l}\text { Физическое функциониро- } \\
\text { вание }(\mathrm{PH}), \mathrm{r}_{\mathrm{s}}\end{array}$ & $\begin{array}{c}0,71 \\
\mathrm{p}<0,0001\end{array}$ & $\begin{array}{c}0,31 \\
\mathrm{p}=0,0004\end{array}$ & $\begin{array}{c}0,29 \\
\mathrm{p}=0,0009\end{array}$ & $\begin{array}{c}0,82 \\
\mathrm{p}<0,0001\end{array}$ & $\begin{array}{c}0,78 \\
\mathrm{p}<0,0001\end{array}$ & $\begin{array}{c}0,79 \\
\mathrm{p}<0,0001\end{array}$ \\
\hline $\begin{array}{l}\text { Ролевое физическое } \\
\text { функционирование (RP), } \mathrm{r}_{\mathrm{s}}\end{array}$ & $\begin{array}{c}0,73 \\
\mathrm{p}<0,0001\end{array}$ & $\begin{array}{c}0,28 \\
\mathrm{p}=0,0009\end{array}$ & $\begin{array}{c}0,35 \\
\mathrm{p}=0,0003\end{array}$ & $\begin{array}{c}0,83 \\
\mathrm{p}<0,0001\end{array}$ & $\begin{array}{c}0,72 \\
\mathrm{p}<0,0001\end{array}$ & $\begin{array}{c}0,88 \\
\mathrm{p}<0,0001\end{array}$ \\
\hline Боль (ВР), $\mathrm{r}_{\mathrm{s}}$ & $\begin{array}{c}0,60 \\
\mathrm{p}<0,0001\end{array}$ & $\begin{array}{c}0,17 \\
\mathrm{p}=0,0113\end{array}$ & $\begin{array}{c}0,08 \\
\mathrm{p}=0,0418\end{array}$ & $\begin{array}{c}0,38 \\
\mathrm{p}=0,0002\end{array}$ & $\begin{array}{c}0,79 \\
\mathrm{p}<0,0001\end{array}$ & $\begin{array}{c}0,32 \\
\mathrm{p}=0,0004\end{array}$ \\
\hline Общее здоровье $(\mathrm{GH}), \mathrm{r}_{\mathrm{s}}$ & $\begin{array}{c}0,82 \\
\mathrm{p}<0,0001\end{array}$ & $\begin{array}{c}0,10 \\
\mathrm{p}=0,0248\end{array}$ & $\begin{array}{c}0,11 \\
\mathrm{p}=0,0211\end{array}$ & $\begin{array}{c}0,89 \\
\mathrm{p}<0,0001\end{array}$ & $\begin{array}{c}0,79 \\
p<0,0001\end{array}$ & $\begin{array}{c}0,40 \\
\mathrm{p}<0,0001\end{array}$ \\
\hline Жизнеспособность (VT), $\mathrm{r}_{\mathrm{s}}$ & $\begin{array}{c}0,76 \\
\mathrm{p}<0,0001\end{array}$ & $\begin{array}{c}0,39 \\
\mathrm{p}=0,0003\end{array}$ & $\begin{array}{c}0,20 \\
\mathrm{p}=0,0015\end{array}$ & $\begin{array}{c}0,58 \\
\mathrm{p}<0,0001\end{array}$ & $\begin{array}{c}0,72 \\
\mathrm{p}<0,0001\end{array}$ & $\begin{array}{c}0,29 \\
\mathrm{p}=0,0009\end{array}$ \\
\hline $\begin{array}{l}\text { Социальное функциониро- } \\
\text { вание (SF), } \mathrm{r}_{\mathrm{s}}\end{array}$ & $\begin{array}{c}0,65 \\
\mathrm{p}<0,0001\end{array}$ & $\begin{array}{c}0,42 \\
\mathrm{p}<0,0001\end{array}$ & $\begin{array}{c}0,12 \\
p=0,0210\end{array}$ & $\begin{array}{c}0,44 \\
\mathrm{p}<0,0001\end{array}$ & $\begin{array}{c}0,39 \\
\mathrm{p}=0,0004\end{array}$ & $\begin{array}{c}0,69 \\
\mathrm{p}<0,0001\end{array}$ \\
\hline $\begin{array}{l}\text { Ролевое эмоциональное } \\
\text { функционирование (RE), } \mathrm{r}_{\mathrm{s}}\end{array}$ & $\begin{array}{c}0,57 \\
\mathrm{p}<0,0001\end{array}$ & $\begin{array}{c}0,24 \\
\mathrm{p}=0,0011\end{array}$ & $\begin{array}{c}0,11 \\
\mathrm{p}=0,0207\end{array}$ & $\begin{array}{c}0,32 \\
p=0,0003\end{array}$ & $\begin{array}{c}0,51 \\
\mathrm{p}<0,0001\end{array}$ & $\begin{array}{c}0,62 \\
\mathrm{p}<0,0001\end{array}$ \\
\hline $\begin{array}{l}\text { Психическое здоровье } \\
\text { (МТ), } \mathrm{r}_{\mathrm{s}}\end{array}$ & $\begin{array}{c}0,50 \\
p<0,0001\end{array}$ & $\begin{array}{c}0,11 \\
\mathrm{p}=0,0199\end{array}$ & $\begin{array}{c}0,22 \\
\mathrm{p}=0,0013\end{array}$ & $\begin{array}{c}0,38 \\
\mathrm{p}=0,0007\end{array}$ & $\begin{array}{c}0,59 \\
p<0,0001\end{array}$ & $\begin{array}{c}0,45 \\
\mathrm{p}=0,0003\end{array}$ \\
\hline $\begin{array}{l}\text { Фактический } \\
\text { биологический возраст } \\
\text { (метод В.П. Войтенко), } \mathrm{r}_{\mathrm{s}}\end{array}$ & $\begin{array}{c}-0,40 \\
\mathrm{p}<0,0001\end{array}$ & $\begin{array}{c}-0,87 \\
\mathrm{p}<0,0001\end{array}$ & $\begin{array}{c}-0,11 \\
\mathrm{p}=0,0211\end{array}$ & $\begin{array}{c}-0,30 \\
p=0,0003\end{array}$ & $\begin{array}{c}-0,54 \\
\mathrm{p}<0,0001\end{array}$ & $\begin{array}{c}-0,30 \\
\mathrm{p}=0,0005\end{array}$ \\
\hline $\begin{array}{l}\text { Количество } \\
\text { сопутствующих } \\
\text { заболеваний, } \mathrm{r}_{\mathrm{s}} \\
\end{array}$ & $\begin{array}{c}0,71 \\
\mathrm{p}<0,0001\end{array}$ & $\begin{array}{c}0,51 \\
\mathrm{p}<0,0001\end{array}$ & $\begin{array}{c}0,19 \\
\mathrm{p}=0,0016\end{array}$ & $\begin{array}{c}0,23 \\
\mathrm{p}=0,0012\end{array}$ & $\begin{array}{c}0,89 \\
\mathrm{p}<0,0001\end{array}$ & $\begin{array}{c}0,41 \\
\mathrm{p}<0,0001\end{array}$ \\
\hline Уровень АДс, $r_{s}$ & $\begin{array}{c}0,4 \\
\mathrm{p}<0,0001\end{array}$ & $\begin{array}{c}0,1 \\
\mathrm{p}=0,0211\end{array}$ & $\begin{array}{c}0,89 \\
\mathrm{p}<0,0001\end{array}$ & $\begin{array}{c}0,64 \\
\mathrm{p}<0,0001\end{array}$ & $\begin{array}{c}0,32 \\
\mathrm{p}=0,0003\end{array}$ & $\begin{array}{c}0,41 \\
\mathrm{p}<0,0001\end{array}$ \\
\hline Уровень ОХС, $\mathrm{r}_{\mathrm{s}}$ & $\begin{array}{c}0,4 \\
\mathrm{p}<0,0001\end{array}$ & $\begin{array}{c}0,12 \\
p=0,0203\end{array}$ & $\begin{array}{c}0,78 \\
p<0,0001\end{array}$ & $\begin{array}{c}0,52 \\
p<0,0001\end{array}$ & $\begin{array}{c}0,21 \\
\mathrm{p}=0,0084\end{array}$ & $\begin{array}{c}0,11 \\
p=0,0210\end{array}$ \\
\hline Толерантность к ФН, $\mathrm{r}_{\mathrm{s}}$ & $\begin{array}{c}0,6 \\
\mathrm{p}<0,0001\end{array}$ & $\begin{array}{c}0,2 \\
p=0,009\end{array}$ & $\begin{array}{c}0,58 \\
p<0,0001\end{array}$ & $\begin{array}{c}0,87 \\
p<0,0001\end{array}$ & $\begin{array}{c}0,41 \\
p<0,0001\end{array}$ & $\begin{array}{c}0,76 \\
p<0,0001\end{array}$ \\
\hline
\end{tabular}

Таблица 3

Результаты анализа сопряженности качественного признака, отражающего степень полиморбидности, с внешними качественными критериями

\begin{tabular}{|l|c|c|c|}
\hline \multicolumn{1}{|c|}{ Качественные критерии } & $\chi^{2}$ & $\begin{array}{c}\text { Значение } \\
\text { достигнутого уровня } \\
\text { значимости «р» }\end{array}$ & $\begin{array}{c}\text { Величина } \\
\text { V-критерия } \\
\text { Крамера }\end{array}$ \\
\hline Пол & 4,21 & 0,12 & 0,20 \\
\hline ОНМК или ТИА в анамнезе & 23,18 & $<0,0001$ & 0,46 \\
\hline Стадия ГБ & 54,76 & $<0,0001$ & 0,50 \\
\hline ПИКС & 23,25 & $<0,0001$ & 0,46 \\
\hline ФК СН & 16,33 & $<0,0001$ & 0,27 \\
\hline Стадия ХСН & 51,90 & $<0,0001$ & 0,49 \\
\hline Наличие тяжелых осложнений СД & 26,05 & $<0,0001$ & 0,34 \\
\hline Стадия ХБП & 32,75 & $<0,0001$ & 0,39 \\
\hline Степень тяжести заболеваний дыхательной системы & 33,82 & $<0,0001$ & 0,41 \\
\hline $\begin{array}{l}\text { Степень тяжести заболеваний костно-мышечной } \\
\text { и соединительной ткани }\end{array}$ & 18,38 & $<0,0001$ & 0,29 \\
\hline
\end{tabular}


Были исследованы сопряженности градации ИП для экспресс-анализа определения степени стойкой нетрудоспособности и степень стойкой нетрудоспособности, установленная у пациентов по результатам медико-социальной экспертизы $\left(\chi^{2}=80,21, \mathrm{p}<0,0001\right)$; градации ИП для определения прогноза результата лечения и прогноз результата лечения, сделанный лечащим врачом $\left(\chi^{2}=59,25, \mathrm{p}<0,0001\right)$; градации ИП для определения риска оперативных вмешательств и риск оперативных вмешательств согласно Lee Index $\left(\chi^{2}=96,37, p<0,0001\right)$; градации ИП для определения показаний к санаторно-курортному лечению и показания к санаторно-курортному лечению согласно Приказу Минздравсоцразвития России $\left(\chi^{2}=205,18, p<0,0001\right)[3]$.

Выявлена статистически значимая взаимосвязь степеней прогностических признаков, опре- деленных посредством МКОП и общепринятыми методами. По интенсивности связь находится на высоком уровне (V-критерий Крамера в диапазоне $0,52-0,97)$, что является доказательством прогностической критериальной В МКОП.

По ряду качественных признаков (пол, стадия ГБ, стадия ХСН, наличие ОНМК или ТИА, ИМ в анамнезе) достигнутые уровни значимости критерия хи-квадрат Пирсона у сформированных для оценки чувствительности МКОП групп имели значения 5\% и более, таким образом, обе группы были однородны по этим признакам и сопоставимы. Не было установлено статистически значимых различий между группами при сравнении медиан следующих количественных признаков: возраст, ТФН на первом этапе исследования, что подтверждает их сопоставимость (таблица 4).

Таблица 4

Результаты сравнения медиан количественных признаков в группах, сформированных для оценки чувствительности МКОП

\begin{tabular}{|c|c|c|c|c|}
\hline \multirow[b]{2}{*}{ Характеристики } & \multirow[b]{2}{*}{$\begin{array}{l}\text { I группа, } \\
\text { n=29 }\end{array}$} & \multirow{2}{*}{$\begin{array}{c}\text { II группа } \\
\text { (контроль), } \\
\text { n=32 }\end{array}$} & \multicolumn{2}{|c|}{$\begin{array}{c}\text { Уровень значимости } \\
\text { «р»» }\end{array}$} \\
\hline & & & $\begin{array}{c}\text { Критерий } \\
\text { Краскела- } \\
\text { Уоллиса }\end{array}$ & $\begin{array}{c}\text { Критерий } \\
\text { Ван дер } \\
\text { Вардена }\end{array}$ \\
\hline Возраст на I этапе, M (Q1-Q3), лет & $56(54-62)$ & $57(53-64)$ & 0,8670 & 0,9090 \\
\hline Толерантность к ФН на I этапе, METs & $5,0(4,5-5,5)$ & $5,1(4,7-5,2)$ & 0,9312 & 0,8859 \\
\hline Толерантность к ФН на II этапе, METs & $7,1(6,7-8,3)$ & $5,7(4,9-5,5)$ & 0,0064 & 0,0102 \\
\hline Индекс полиморбидности на I этапе, у.е. & $\begin{array}{c}0,71 \\
(0,62-0,78)\end{array}$ & $0,69(0,59-0,80)$ & 0,4568 & 0,3294 \\
\hline Индекс полиморбидности на II этапе, у.е. & $\begin{array}{c}0,81 \\
(0,71-0,89)\end{array}$ & $0,74(0,66-0,87)$ & 0,0341 & 0,0289 \\
\hline $\begin{array}{l}\text { Индекс кластера немодифицируемых } \\
\text { индикаторов на I этапе, у.е. }\end{array}$ & $\begin{array}{c}0,69 \\
(0,61-0,75)\end{array}$ & $0,71(0,62-0,76)$ & 0,4787 & 0,2684 \\
\hline $\begin{array}{l}\text { Индекс кластера немодифицируемых } \\
\text { индикаторов на II этапе, у.е. }\end{array}$ & $\begin{array}{c}0,68 \\
(0,61-0,74)\end{array}$ & $0,70(0,61-0,76)$ & 0,3985 & 0,2057 \\
\hline $\begin{array}{l}\text { Индекс кластера модифицируемых индикаторов } \\
\text { на I этапе, у.е. }\end{array}$ & $\begin{array}{c}0,69 \\
(0,57-0,74)\end{array}$ & $0,70(0,62-0,75)$ & 0,2565 & 0,1248 \\
\hline $\begin{array}{l}\text { Индекс кластера модифицируемых индикаторов } \\
\text { на II этапе, у.е. }\end{array}$ & $\begin{array}{c}0,82 \\
(0,79-0,87)\end{array}$ & $0,72(0,65-0,80)$ & 0,0121 & 0,0117 \\
\hline $\begin{array}{l}\text { Индекс кластера коморбидной патологии на I } \\
\text { этапе, у.е. }\end{array}$ & $\begin{array}{c}0,58 \\
(0,56-0,67)\end{array}$ & $0,57(0,55-0,66)$ & 0,3451 & 0,4228 \\
\hline $\begin{array}{l}\text { Индекс кластера коморбидной патологии на II } \\
\text { этапе, у.е. }\end{array}$ & $\begin{array}{c}0,59 \\
(0,57-0,68)\end{array}$ & $0,59(0,57-0,66)$ & 0,6687 & 0,4254 \\
\hline $\begin{array}{l}\text { Индекс кластера мультиморбидной патологии на } \\
\text { I этапе, у.е. }\end{array}$ & $\begin{array}{c}0,63 \\
(0,59-0,73)\end{array}$ & $0,65(0,60-0,76)$ & 0,0845 & 0,0625 \\
\hline $\begin{array}{l}\text { Индекс кластера мультиморбидной патологии на } \\
\text { II этапе, у.е. }\end{array}$ & $\begin{array}{c}0,75 \\
(0,68-0,79)\end{array}$ & $0,69(0,65-0,74)$ & 0,0289 & 0,0194 \\
\hline Индекс функционального кластера на I этапе, у.е. & $\begin{array}{c}0,64 \\
(0,53-0,71)\end{array}$ & $0,66(0,56-0,74)$ & 0,1598 & 0,1167 \\
\hline $\begin{array}{l}\text { Индекс функционального кластера на II этапе, } \\
\text { у.е. }\end{array}$ & $\begin{array}{c}0,80 \\
(0,79-0,87)\end{array}$ & $0,70(0,63-0,77)$ & 0,0091 & 0,0057 \\
\hline
\end{tabular}


Оценка чувствительности МКОП для ее индексов путем вычисления индекса GRI

\begin{tabular}{|c|c|}
\hline Индексы МКОП & Индекс GRI \\
\hline Индекс полиморбидности (ИП) & $-0,81$ \\
\hline Индекс кластера немодифицируемых индикаторов (И1) & $-0,19$ \\
\hline Индекс кластера модифицируемых индикаторов (И2) & $-3,92$ \\
\hline Индекс кластера коморбидной патологии (И3) & $-0,21$ \\
\hline Индекс кластера мультиморбидной патологии (И4) & $-1,18$ \\
\hline Индекс функционального кластера (И5) & $-2,24$ \\
\hline
\end{tabular}

На первом этапе исследования группы были статистически однородны при сравнении медиан как ИП, так и промежуточных индексов МКОП. Через 6 месяцев диспансерного наблюдения установлено статистически значимое различие между группами при сравнении медиан следующих количественных признаков: ТФН, ИП, И2, И4, И5. Таким образом, подтверждается чувствительность ИП, И2, И4 и И5 к изменениям ТФН у пациентов. Медианы И1 и И3 через 6 месяцев диспансерного наблюдения остались статистически однородны в первой и второй группах, что объясняется содержащимися в этих кластерах индикаторами, не способными значимо изменять свои значения с течением времени.

Индексы чувствительности Guyatt Responsiveness Index (GRI) [10] были вычислены для ИП и промежуточных индексов кластеров МКОП на первом (до начала ДН) и третьем (через 6 месяцев ДН) этапах исследования (таблица 5).

Полученные значения индекса чувствительности GRI колебались в диапазоне от 0,19 до 3,92. И1 и ИЗ показали незначительную чувствительность к изменениям в состоянии здоровья пациентов. Остальные индексы МКОП показали чувствительность средней (ИП, И4) и высокой интенсивности (И2, И5).

Таким образом, валидация МКОП доказала ее надежность (коэффициент Кронбаха $\alpha$ превысил 0,70 , установлена умеренная или сильная степень корреляции индексов МКОП методом «тестретест»). На основании статистического подтверждения гипотез о наличии и отсутствии различных по интенсивности корреляций индексов МКОП и сопряженностей степени полиморбидности с внешними критериями доказана конвергентная и дискриминативная критериальная валидность. Конкурентная валидность была доказана наличием сильной обратной связи (коэффициент корреляции Спирмена составил от $-0,83$ до $-0,91$ при уровне значимости $\mathrm{p}<0,0001)$ результатов оценки П посредством МКОП и результатов других наиболее распространенных методик ее оценки (индекс Kaplan-Feinstein, система CIRS-G, индекс Charlson). Выявлена статистически значи- мая взаимосвязь степеней прогностических признаков, определенных посредством МКОП и общепринятыми методами. По интенсивности связь находится на высоком уровне ( $\mathrm{V}$-критерий Крамера в диапазоне 0,52-0,97), что является доказательством прогностической критериальной В МКОП. Чувствительность МКОП была доказана путем расчета предсказуемых различий ее индексов. Между сформированными для доказательства чувствительности группами установлено ожидаемое статистически значимое различие при сравнении медиан большинства индексов МКОП. Полученные для них значения индекса чувствительности GRI колебались в диапазоне от $-0,81$ до $-3,92$ и показали чувствительность средней и высокой интенсивности.

Индексы и кластеры МКОП высокодифференцированы и отражают соответствующую их названию информацию.

МКОП рекомендуется к использованию в медицинских организациях, оказывающих ПМСП, с целью оптимизации и совершенствования лечебно-диагностической, реабилитационной, экспертной и профилактической работы с пациентами, имеющими метаболический синдром на фоне полиморбидной патологии.

\section{ЛИТЕРАТУРА / REFERENCES}

1. Войтенко В.П. Биологический возраст // Биология старения.- Л. : Наука, 1982. - С. 102-115. [Voyten$k o$ V.P. Biological age. in Biology of aging. L. : Nauka; 1982: 102-115 (in Russ.)].

2. ГОСТ Р 52379-2005 Надлежащая клиническая практика. - М. : Стандартинформ, 2006. - 39 с. [GOST R 52379-2005 Good clinical practice. M. : Standartinform; 2006: 39 s. (in Russ.)].

3. Приказ Минздравсоцразвития России от 22.11.2004 № 256 (ред. от 15.12.2014) «О Порядке медицинского отбора и направления больных на санаторно-курортное лечение» (зарегистрировано в Минюсте России 14.12.2004 № 6189). [Order of the Ministry of Health and Social Development of Russia from 22.11.2004 № 256 (Edited on December 15, 2014) "On the Procedure for Medical Selection and Referral of Patients for Sanatorium and Spa Treat- 
ment" (registered in the Ministry of Justice of Russia on December 14, 2004 No. 6189) (in Russ.)].

4. Приказ Министерства здравоохранения Российской Федерации от 3 февраля 2015 г. № 36н «Об утверждении порядка проведения диспансеризации определенных групп взрослого населения». [Order of the Ministry of Health of the Russian Federation of February 3, 2015, No. 36n, "On Approval of the Procedure for Clinical Screening of Certain Adult Populations." (in Russ.)].

5. Приказ Министерства здравоохранения РФ от 21 декабря 2012 г. № 1344н «Об утверждении Порядка проведения диспансерного наблюдения». [Order of the Ministry of Health of the Russian Federation from December 21, 2012 № 1344H "On approval of the procedure for dispensary observation." (in Russ.)].

6. Чазова И.Е., Мычка В.Б., Кисляк О.А., Кузнечова И.В., Литвин А.Ю., Шестакова М.В., Бугрова С.А., Звенигородская Л.А., Кошельская О.А., Кухарчук В.В., Мамедов М.Н.О., Медведева И.В., Мкртумян А.М., Небиеридзе Д.В., Недогода С.В., Перепечь Н.Б., Подзолков В.И., Симонова Г.И., Титов В.Н., Тюрина Т.В., Фурсов А.Н., Хирманов В.Н., Чукаева И.И., Шальнова С.А., Шубина А.Т., Всероссийское научное общество кардиологов, Российское медииинское общество по артериальной гипертонии. Диагностика и лечение метаболического синдрома. Российские рекомендации (второй пересмотр) // Кардиоваскулярная терапия и профилактика. - 2009. - Т. 8, № 6, Приложение 2. - C. 1-29. [Chazova I.E., Mychka V.B., Kislyak O.A., Kuznetsova I.V., Litvin A.Yu., Shestakova M.V., Bugrova S.A., Zvenigorodskaya L.A., Koshel'skaya O.A., Kukharchuk V.V., Mamedov M.N.O., Medvedeva I.V., Mkrtumyan A.M., Nebiyeridze D.V., Nedogoda S.V., Perepech' N.B., Podzolkov V.I., Simonova G.I., Titov V.N., Tyurina T.V., Fursov A.N., Khirmanov V.N., CHukayeva I.I., Shal'nova S.A., Shubina A.T., Vserossiyskoye nauchnoye obshchestvo kardiologov, Rossiyskoye meditsinskoye obshchestvo po arterial'noy gipertonii. Diagnosis and treatment of metabolic syndrome. Russian recommendations (second revision). Kardiovaskulyarnaya terapiya i profilaktika. 2009; 8 (6, Suppl. 2): 1-29 (in Russ.)].
7. Charlson M.E., Pompei P., Ales H.L., MacKenzie C.R. A new method of classifying prognostic comorbidity in longitudinal studies: Development and validation // Journal Chronic Disease. - 1987. - Vol. 40, N 5. P. 373-383.

8. Cronbach L.J. Coefficient alpha and the internal structure of tests // Psychometrika. - 1951. - Vol. 16, N 3. - P. 297-334.

9. Fagerstrom $K$. Determinants of tobacco use and renaming the FTND to the Fagerstrom Test for Cigarette Dependence // Nicotine Tob. Res. - 2012. - Vol. 14, N 1. - P. 75-78 - DOI:10.1093/ntr/ntr137.

10. Guyatt G., Walter S., Norman G. Measuring Change over Time: Assessing the Usefulness of Evaluative Instruments // J. Chronic. Dis. - 1987. - Vol. 40, N 2. P. 171-178.

11. Hlatky M.A., Boineau R.E., Higginbotham M.B., Lee K.L., Mark D.B., Cliff R.M., Cobb F.R., Pryor D.B A brief selfadministered questionnaire to determine functional capacity (the Duke Activity Status Index) // Am J Cardiol. - 1989. - Vol. 64, N 10. - P. 651-654.

12. Kaplan M.H., Feinstein A.R. Acritique of methods in reported studies of long-term vascular complications in patients with diabetes mellitus // Diabetes. 1973. - Vol. 22, N 3. - P. 160-174.

13. Lee T.H., Marcantonio E.R., Mangione C.M., Thomas E.J., Polanczyk C.A., Cook E.F., Sugarbaker D.J., Donaldson M.C., Poss R., Ho K.K., Ludwig L.E., Pedan A., Goldman L. Derivation and prospective validation of a simple index for prediction of cardiac risk of major noncardiac surgery // Circulation. - 1999. Vol. 100, N 10. - P. 1043-1049.

14. Miller M.D., Towers A. Manual of Guidelines for Scoring the Cumulative Illness Rating Scale for Geriatrics (CIRS-G). - Pittsburg, Pa : University of Pittsburgh., 1991. - 31 p.

15. Ware J.E., Snow K.K., Kosinski M., Gandek B. SF-36 Health Survey. Manuel and Interpretation Guide. Lincoln, RI. : Quality Metric Incorporated, 2000. $150 \mathrm{p}$.

16. Zigmond A.S., Snaith R.P. The hospital anxiety and depression scale // Acta Psychiatrica Scandinavica. 1983. - Vol. 67, N 6. - P. 361-370. 